\title{
The Broad Applicability of Memory Bias and Its Coexistence With the Planning Fallacy: Reply to Griffin and Buehler (2005)
}

\author{
Michael M. Roy, Nicholas J. S. Christenfeld, and Craig R. M. McKenzie \\ University of California, San Diego
}

\begin{abstract}
People chronically underestimate how long tasks will take. In their original article, the present authors (M. M. Roy, N. J. S. Christenfeld, \& C. R. M. McKenzie, 2005) suggested a simple, broadly applicable explanation: Biased predictions result from biased memories. In their comment article, D. Griffin and R. Buehler (2005) suggested that in many domains in which this memory-bias account appears to outpredict their own account, theirs actually makes no prediction at all. However, the present authors did not suggest that only 1 theory is right but that theirs is consistent with data that prior theories, including their own, cannot explain. Ignoring memories of past tasks is not a complete explanation for the phenomenon if the memories people could consult are themselves biased. Nonetheless, underestimating future task duration is almost certainly multiply determined, and thus our account and theirs can coexist.
\end{abstract}

Keywords: planning fallacy, memory bias, prediction, underestimation, time

People chronically underestimate how long tasks, from folding the laundry, to writing a textbook, to tunneling under the English Channel, will take. Though there have been many suggestions offered for this phenomenon, we proposed in our article "Underestimating the Duration of Future Events: Memory Incorrectly Used or Memory Bias?" a simple, broadly applicable, but overlooked explanation: People, when they have just finished a task, are likely to think it took less time than it actually did. Given this, when they try to predict how long it will take next time, they can hardly help but underestimate. Consistent with such an explanation, we found that (a) there is a tendency to underestimate task duration retrospectively; (b) the tendency to underestimate future duration disappears for novel tasks, for which there are no biased memories to consult; (c) variables that affect memory of past duration, such as experience with the task, task duration, and motivation, have a similar effect on predictions; (d) correcting memory of past duration enhances predictive accuracy. Thus, the memory bias account not only explains why people tend to underestimate future task duration, it also leads to predictions of when underestimation is most likely and how best to eliminate underestimation.

Griffin and Buehler (2005) suggested that the aim of our article was to contrast our memory bias account with the planning fallacy. We actually compared the predictive utility of memory bias with a more general model of prediction that takes into account a number of different theories in which memory for past duration is not used (cf. Byram, 1997). The planning fallacy account is a specific instance of the more general model, and, being the most developed, it was the one on which we focused most attention. Further-

Michael M. Roy, Nicholas J. S. Christenfeld, and Craig R. M. McKenzie, Department of Psychology, University of California, San Diego.

Correspondence concerning this article should be addressed to Michael M. Roy, who is now at the Department of Psychology, University of Illinois at Urbana-Champaign, 603 East Daniel Street, Champaign, IL 61820. E-mail: mroy@cyrus.psych.uiuc.edu more, as we pointed out in our original article, the planning fallacy and memory bias accounts may coexist. Our argument is that the memory bias account can explain more of the extant data than can the other theories, including the planning fallacy.

As the planning fallacy has been refined over time, the scope of the theory has narrowed. Griffin and Buehler suggested that many instances of underestimation-including most laboratory tasksare not relevant to their theory. They suggested that it is not underestimation of task duration but optimism about task completion time that is the proper domain of their theory. They suggested furthermore that their theory predicts that, although the error comes from ignoring past durations, reminding people of the distributional information should not enhance predictions. We briefly discuss these notions and offer some general comments about the opposition or compatibility of the two theories.

The core of the planning fallacy is that people take a singular perspective and ignore distributional information about past completion times when predicting duration. This, however, is not sufficient to produce underestimation - it must be combined with theoretically separate mechanisms such as irrational optimism or the forgetting of subcomponents - and it is also hard to reconcile with the estimates being close to reality, albeit systematically biased. If memory for task duration, at least in some form, is not consulted, there is no reason to believe that estimates based on a singular perspective would have any predictive validity. It takes less time to sharpen a pencil than to write a book with it, and people, with some sense of how long things generally take, will unerringly predict so. Predictions are not indiscriminately optimistic (Armor \& Taylor, 1998), and there is a strong relationship between predicted and actual duration when comparison is made across participants (Buehler et al., 1994). To deal with this, one has to allow people to incorporate some memories of past times in their predictions. One would naturally think that making those memories more salient would give them more weight and hence, if the planning fallacy account is correct, reduce underestimation. However, Griffin and Buehler suggest that their theory predicts no 
such effect and is thus consistent with findings showing the ineffectiveness of reminding people of their past experiences with the task. It would be helpful if the planning fallacy was more specific about what, if any, role memory plays in prediction and how a fairly accurate prediction of duration could be made if memory, whether for task duration or task completion time, is not consulted.

Griffin and Buehler make much of the distinction between predictions of task duration and time of completion. However, we suggest that underestimating task duration and missing deadlines can be driven by the same underlying problem. When one finds oneself still struggling with a task long after one had expected it to be done, it is likely because it took longer than one expected or other necessary but interfering tasks themselves took longer than expected.

It strikes us as an odd requirement that one must know, when people make a prediction for the duration of a task, whether they are relatively pessimistic about that task before one can tell whether the planning fallacy applies and thus whether their estimate is likely to be too short. Our approach suggests that if the task is at least reasonably long, and they are in a position to have some memories of its duration, then, given that those memories are likely to be short, the predictions will also be so. We do not require a separate theory for lab tasks, for uninterrupted tasks, for tasks without general pessimism, and the like.

We are not suggesting that the planning fallacy is wrong or useless. People may well fail to consider the entire historical record. They might sometimes forget task subcomponents. Believing one is smarter or more talented than reality warrants can play a part, too. Our point, however, is that people are likely to underestimate, although possibly less, without these factors playing a role. Griffin and Buehler (2005) concede this point, albeit without admitting it falls outside the domain of their theory. They suggested, in contrast to Kahneman and Tversky's (1979) original suggestion that anything that increases "perceived uniqueness" of the task will exacerbate the planning fallacy, that novelty should have no systematic effect on estimation bias and that the outcome would depend on unpredictable details. Our theory implies, and the data support, a simpler hypothesis: Experience with a task has a similar effect on both memory and prediction. We understand showing that factors that influence memories also influence predictions is not equivalent to showing that the predictions are based on the memories. We discuss this in our article, and the bulk of our attention was devoted to showing the connection is more than a "misleading surface analogy." Again and again, the correspondence between shifts in memory and shifts in prediction was obtained. The theory is logically coherent, parsimonious, and predicts the data. It would be interesting to hear an explanation of how people could fail to underestimate future times, with underestimates being all they have available in memory. Although we agree with Griffin and Buehler that it is important to keep in mind that correlation does not imply causation, the pattern of results needs to be explained. Our theory does that.

We are in agreement with Griffin and Buehler on the need for added empirical work aimed at directly testing the predictions of memory bias. Indeed, in our article we suggested a number of areas in which further research would be useful. We believe that testing the specific predictions of memory bias would be fruitful, and, more importantly, should lead to interventions that would help improve prediction accuracy.

\section{References}

Armor, D. A., \& Taylor, S. E. (1998). Situated optimism: Specific outcome expectancies and self-regulation. In M. P. Zanna (Ed.), Advances in experimental social psychology (Vol. 30, pp. 309-379). San Diego, CA: Academic Press.

Buehler, R., Griffin, D., \& Ross, M. (1994). Exploring the "planning fallacy": Why people underestimate their task completion times. Journal of Personality and Social Psychology, 67, 366-381.

Byram, S. J. (1997). Cognitive and motivational factors influencing time prediction. Journal of Experimental Psychology: Applied, 3, 216-239.

Griffin, D., \& Buehler, R. (2005). Biases and fallacies, memories and predictions: Comment on Roy, Christenfeld, and McKenzie (2005). Psychological Bulletin, 131, 757-760.

Kahneman, D., \& Tversky, A. (1979). Intuitive prediction: Biases and corrective procedures. TIMS Studies in Management Sciences, 12, 313 327.

Roy, M. M., Christenfeld, N. J. S., \& McKenzie, C. R. M. (2005). Underestimating the duration of future events: Memory incorrectly used or memory bias? Psychological Bulletin, 131, 738-756.

Received May 9, 2005

Revision received May 19, 2005

Accepted May 19, 2005 